http://www.jfas.info

\title{
EMPIRICAL MODEL FOR TERENGGANU FORWARD SCATTER RADAR SEASIDE CLUTTER WITH UHF BAND
}

\author{
N. N. Ismail ${ }^{1, *}$, N. E. A. Rashid ${ }^{1,2}$, Z. Ismail $\operatorname{Khan}^{1,2}$ and N. Ripin ${ }^{1}$ \\ ${ }^{1}$ Faculty of Electrical Engineering, Universiti Teknologi MARA, 40450 Shah Alam, Selangor, \\ Malaysia \\ ${ }^{2}$ Applied Electromagnetic Research Group (AERG), Advanced Computing and \\ Communication Communities Research, Universiti Teknologi MARA, 40450 Shah Alam, \\ Selangor, Malaysia
}

Published online: 10 September 2017

\begin{abstract}
An empirical model for Terengganu seaside clutter measured using forward scatter radar (FSR) with operating frequency of $434 \mathrm{MHz}$ is presented in this paper based on the analysed characteristics. Statistical distribution approach is used in this analysis to model the simulated clutter-like signal. To validate the accurateness between the measured and simulated clutter signal, the envelope clutter signal and the number of fit shape factor for both signals are being compared, while root mean square error (RMSE) technique is used to calculate the percentage error between the measured and simulated clutter signal.
\end{abstract}

Author Correspondence, e-mail: nornajwaismail88@gmail.com

doi: http://dx.doi.org/10.4314/jfas.v9i3s.16

\section{INTRODUCTION}

Clutter signal is an unwanted signal collected from the surrounding. The movement of swaying vegetation and foliage is the source of ground radar clutter signal [1]. The presence of clutter signal to the radar system not only limits the performance, but also masks the useful 
target signal and complicates the target detection.

This factor contributes to a false alarm to the radar system [2-3]. The amplitude of the clutter signal varies according to the wind speed. The stronger the wind during the event, the higher the amplitude of the clutter signal. As a result, users may have some difficulties to discriminate between the target signal and the clutter signal. Therefore, in order to eliminate the clutter effect that occurs to the radar system, a clutter model need to be developed. The model can be used to develop effective threshold signal detection as presented in [4], which can improve the performance of the radar system during the clutter event. Besides that, the model also can be used to generate a synthetic forest clutter scene and can be used to improve the factor test [5] and predict radar performance [6-7, 13].

As the clutter signal distributed within a period of time, one of the methods to model a clutter signal is by using a probability distribution function (PDF) approach. This method has been used in previous researches to model the clutter signal for different types of clutter signal with different values of operating frequency [8-11]. This paper is the continuous work to model the clutter signal for the statistical analysis that has been done in Terengganu seaside measured using FSR with $434 \mathrm{MHz}$ as presented in [12].

\section{MEASURED CLUTTER DATA}

The measurement descriptions of the clutter signal for FSR system with $434 \mathrm{MHz}$ at Terengganu seaside was presented in [12]. Fig. 1 shows the measured clutter signal with (a) low, (b) medium, (c) strong and (d) very strong clutter strength.

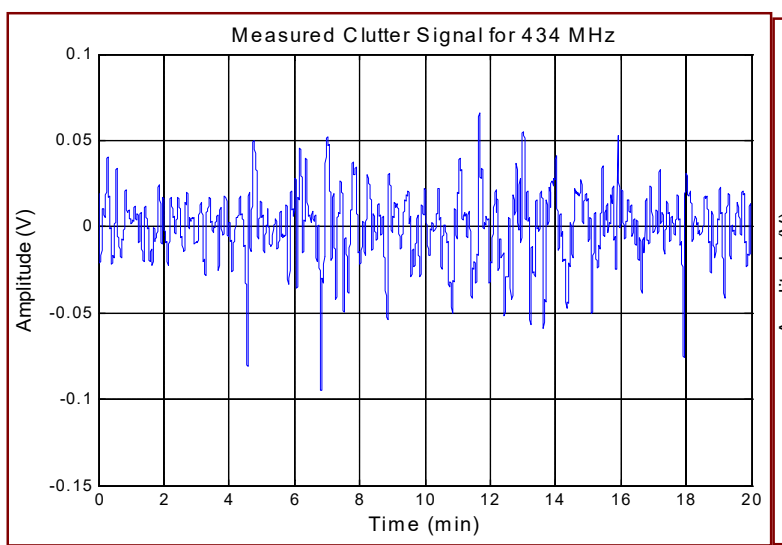

(a)

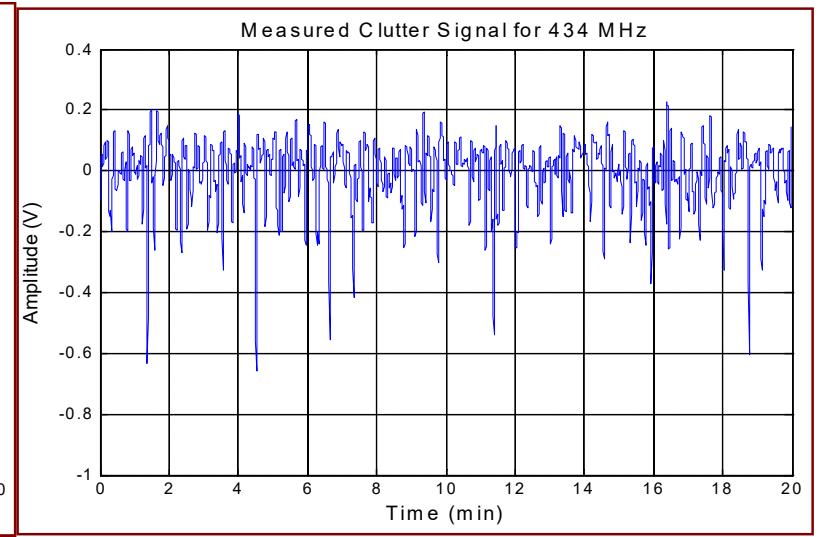

(b) 


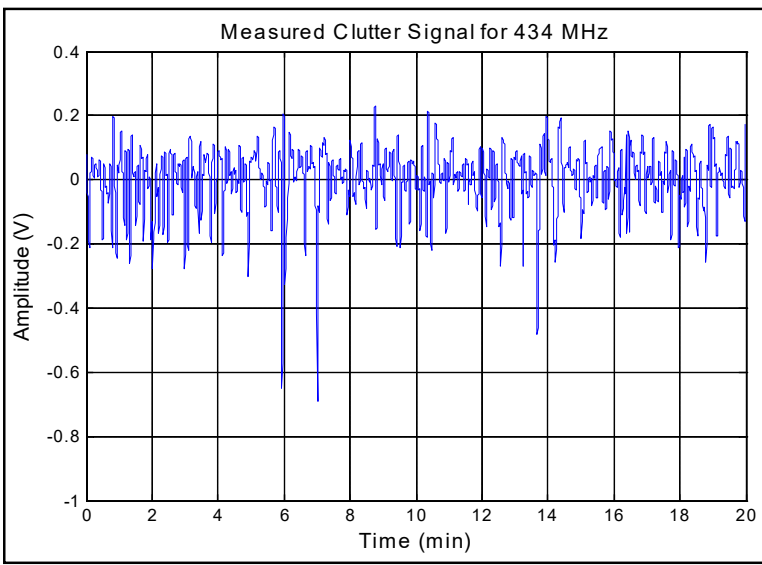

(c)

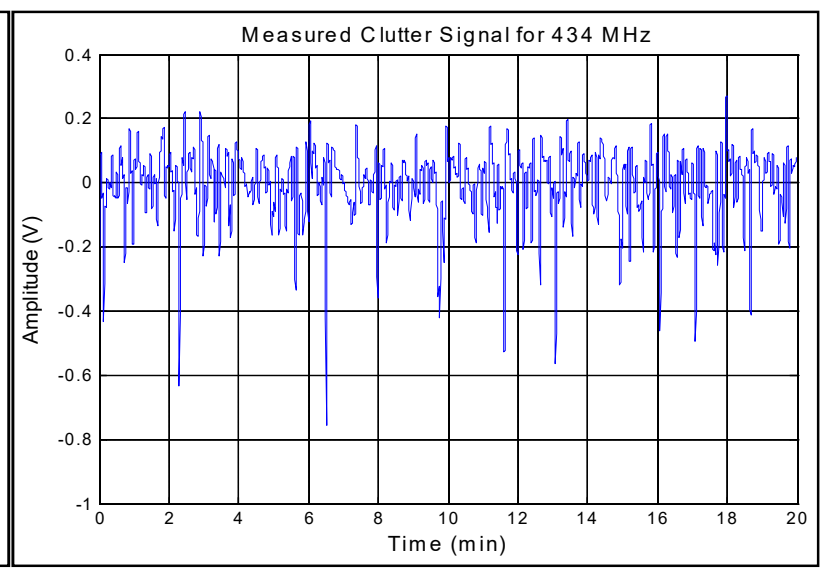

(d)

Fig.1. Measured clutter signal, (a) low, (b) medium, (c) strong, and (d) very strong The main influence to the clutter signal at the seaside is the wind coming from the sea that blow the vegetation along the seaside. As the wind speed increased, the amplitude of the clutter signal become higher. Since the amplitude of the clutter signal is dependent to the wind speed, thus the clutter strength is categorized based on the wind speed condition during the measurement as tabulated in Table 1. Each data was recorded for 20 minutes. These measured clutter data will be used to model the clutter signal based on its characteristics.

Table 1. Wind speed conditions during measurement

\begin{tabular}{cc} 
Wind Condition & Average Wind Speed $(\mathbf{k m} / \mathbf{h})$ \\
\hline Low & $5-10$ \\
Medium & $10-15$ \\
Strong & $15-20$ \\
Very Strong & $>20$ \\
\hline
\end{tabular}

\section{CLUTTER STATISTICAL PROPERTIES}

The measured clutter data properties are tabulated in Table 2. Such properties are the standard deviation of the measured clutter signal, standard deviation of the envelope clutter signal, maximum and minimum of the envelope clutter signal, standard deviation of noise signal and number of fit shape factor. These values will be used in MATLAB software to generate the simulated clutter signal. Based on the previous finding in [12], the distribution models that fits 
best to the measured clutter signal for $434 \mathrm{MHz}$ is Log-Logistic for all clutter strength. Fig. 2 shows the signal distribution of the measured clutter signal with the number of fit shape factor. It is recorded that the shape factor is 0.37 for low and medium clutter while 0.39 for strong and very strong clutter. These shape factor values will be used as the benchmark to generate the simulated clutter signal.

Table 2. Measured Clutter Signal Properties

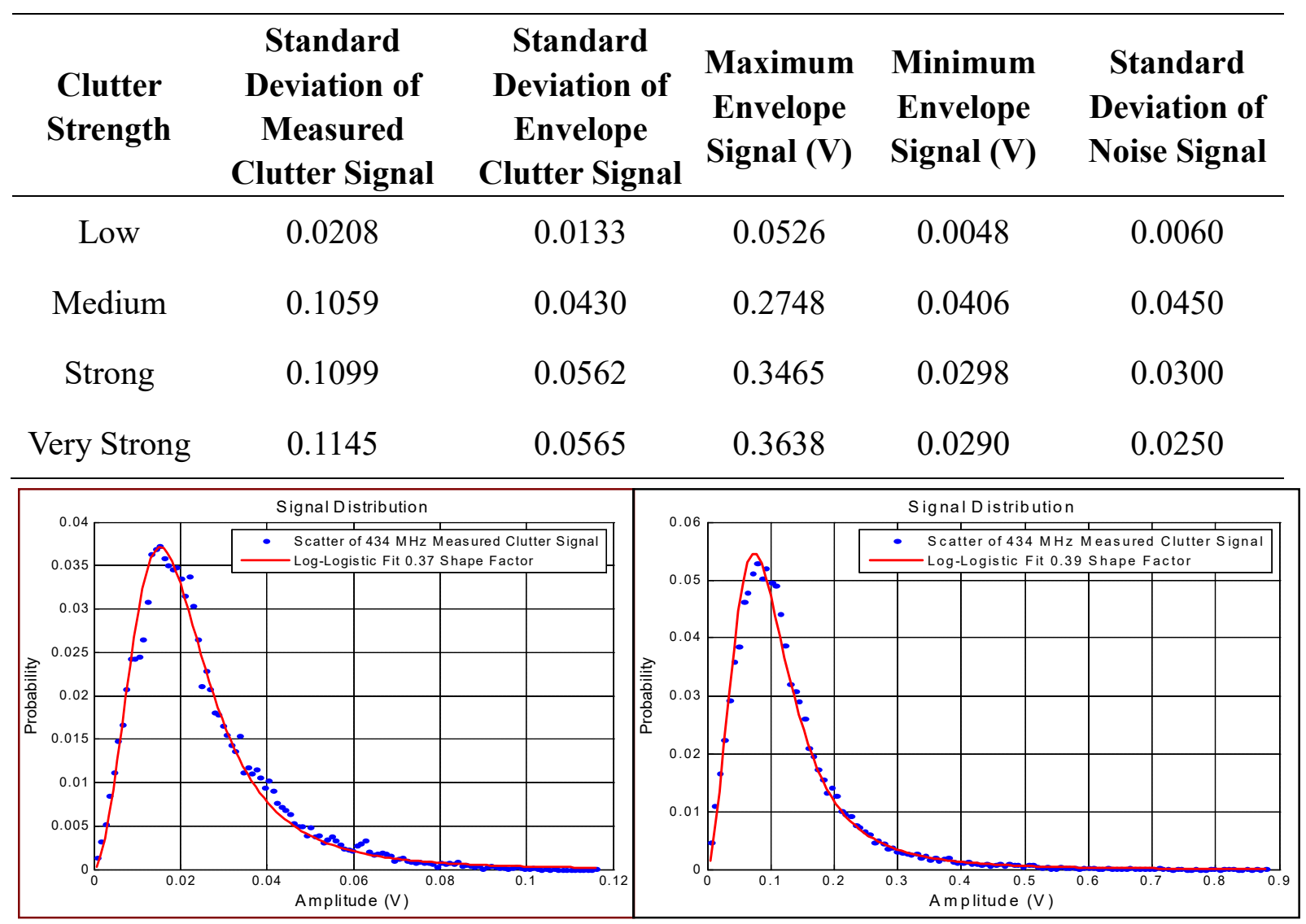

(a)

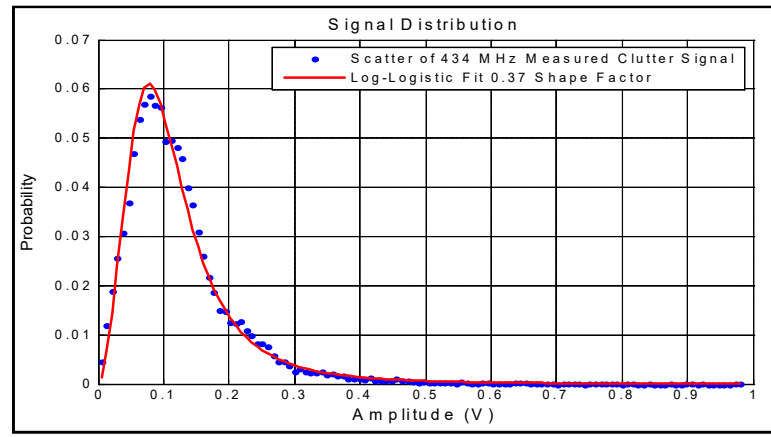

(b)

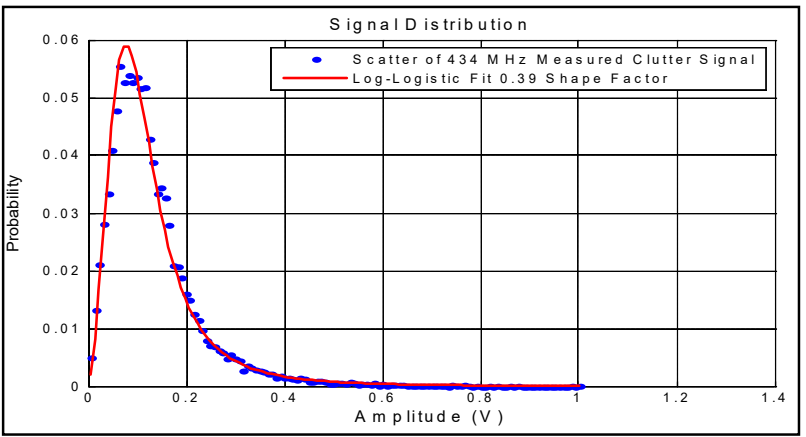

(d)

Fig.2. Signal distribution of measured clutter signal with number of fit shape factor, (a) low, (b) medium, (c) strong, and (d) very strong 


\section{CLUTTER SIMULATION MODEL}

This section consists of five parts, starts with the flow explanation of the clutter simulation, the results of the simulated clutter signal, the comparison of the envelope signal for measured and simulated clutter signal, the signal distribution of the simulated clutter signal with the number of fit shape factor and the percentage error between the measured and simulated clutter signal.

\subsection{Clutter Simulation Model Block Diagram}

In order to generate the simulated clutter signal, there are two main consecutive steps, which are (1) generating the random signal based on the standard deviation of the measured and envelope clutter signal passing through the Butterworth filter and adjustment according to the maximum and minimum envelope signal followed by the modulation process before going through the (2) adding process that requires the generation of random noise signal to be added with modulated signal as shown in Fig. 6.

\subsection{Simulated Clutter Signal}

Since the clutter generation is based on the random signal, therefore it is impossible to produce exactly a clutter signal as the measured clutter signal due the nature from surrounding during the measurement. The simulated clutter signal for $434 \mathrm{MHz}$ is presented in Fig. 3 with (a) low, (b) medium, (c) strong and (d) very strong clutter. The envelope clutter signal comparison between the measured and simulated clutter signal need to be done in order to validate whether the simulated clutter signal is replicating the measured clutter signal. The results are presented in section $\mathrm{C}$.

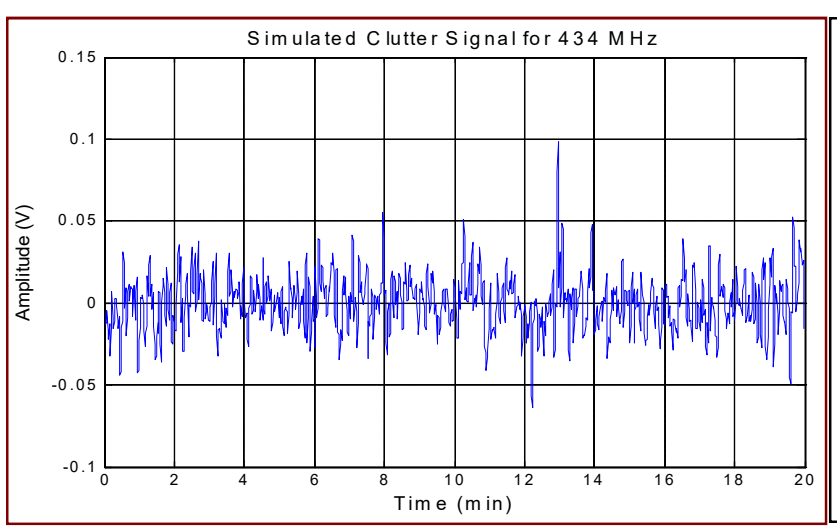

(a)

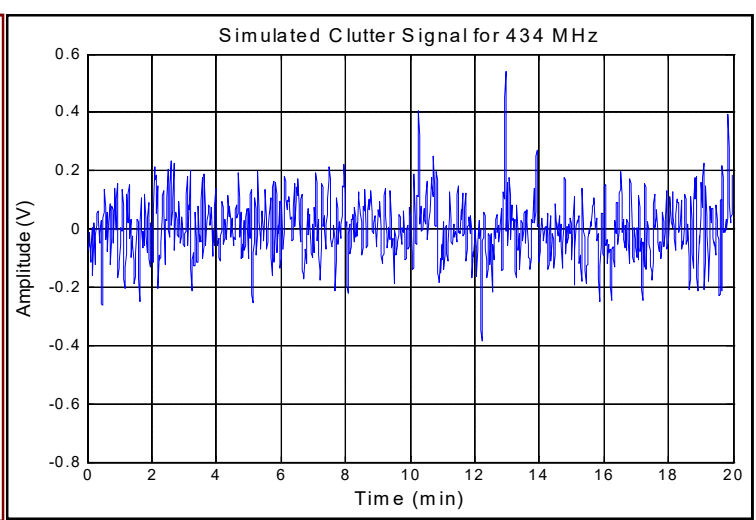

(b) 


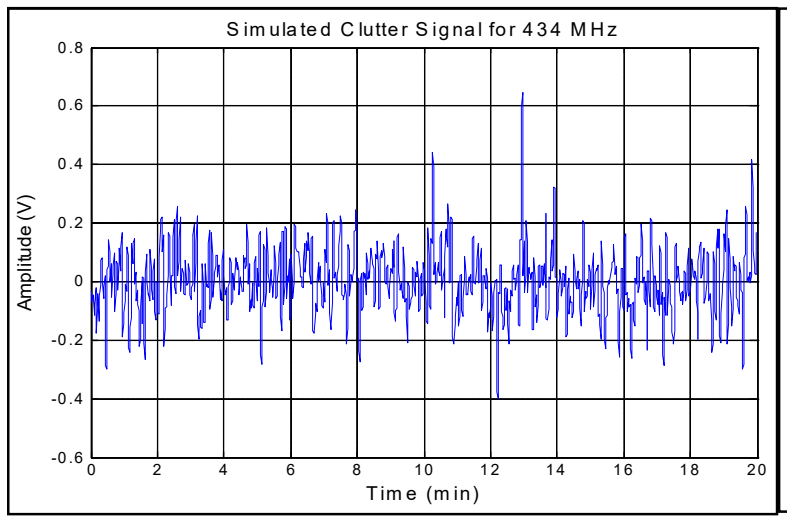

(c)

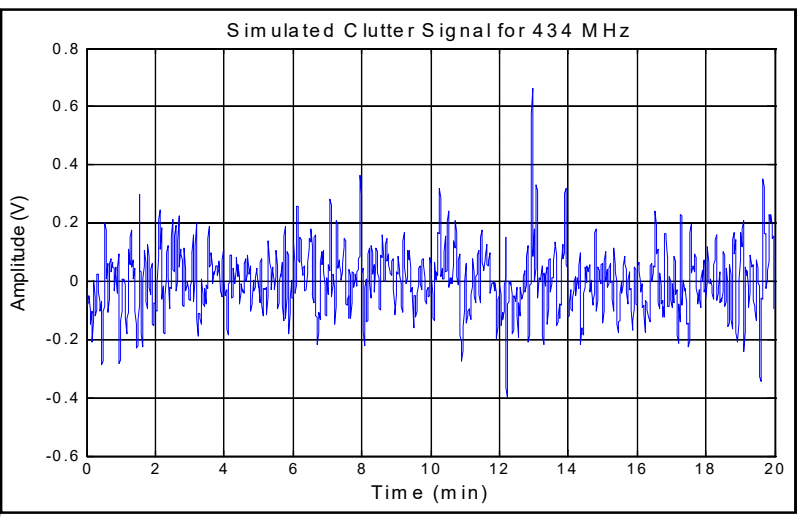

(d)

Fig.3. Simulated clutter signal, (a) low, (b) medium, (c) strong, and (d) very strong

\subsection{Envelope Clutter Signal Comparison}

Fig. 4 shows the envelope clutter signal comparison between the measured and simulated clutter signal for (a) low, (b) medium, (c) strong and (d) very strong clutter strength. From the figures, its show that the simulated clutter signal is quite similar to the measured clutter signal for all clutter strength.

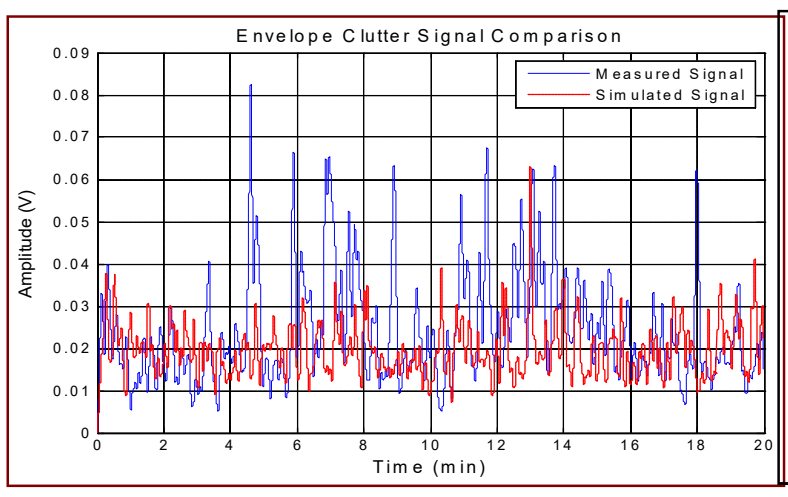

(a)

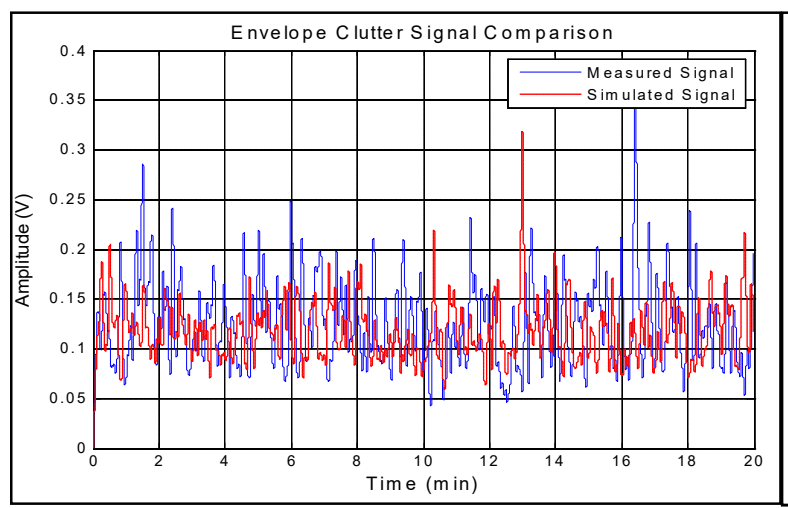

(b)

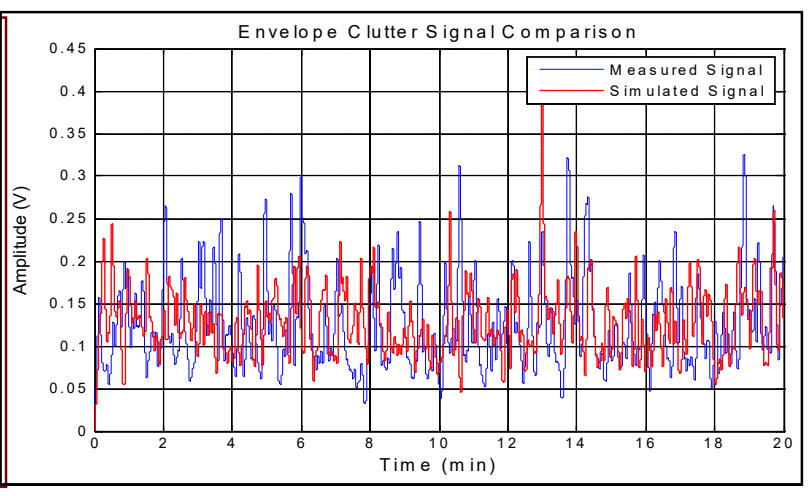

(c)

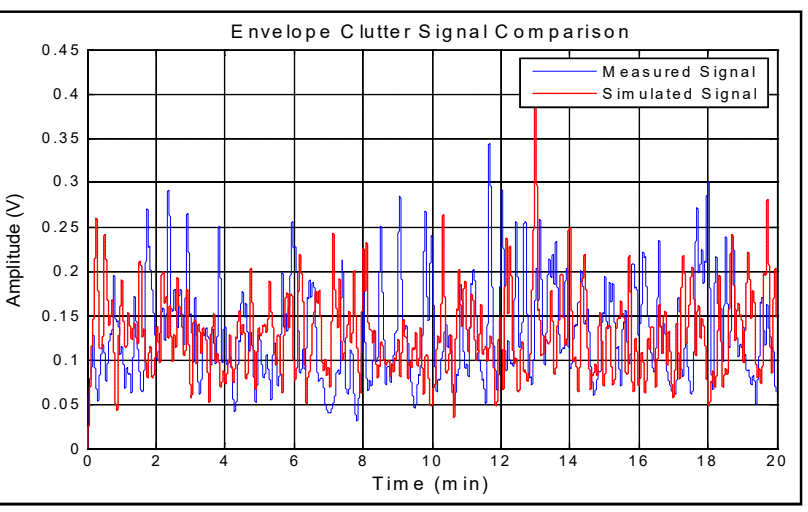

(d)

Fig.4. Envelope clutter signal comparison, (a) low, (b) medium, (c) strong, and (d) very strong 


\subsection{Signal Distribution for Simulated Clutter Signal}

Fig. 5 shows the signal distribution for simulated clutter signal with number of fit factor for (a) low, (b) medium, (c) strong and (d) very strong clutter strength. From the figures, it can be seen that the number of fit shape factor for low and medium clutter is 0.37 , while for strong and very strong clutter is 0.39 which follows the number of fit shape factor for the measured clutter as in Fig. 2.

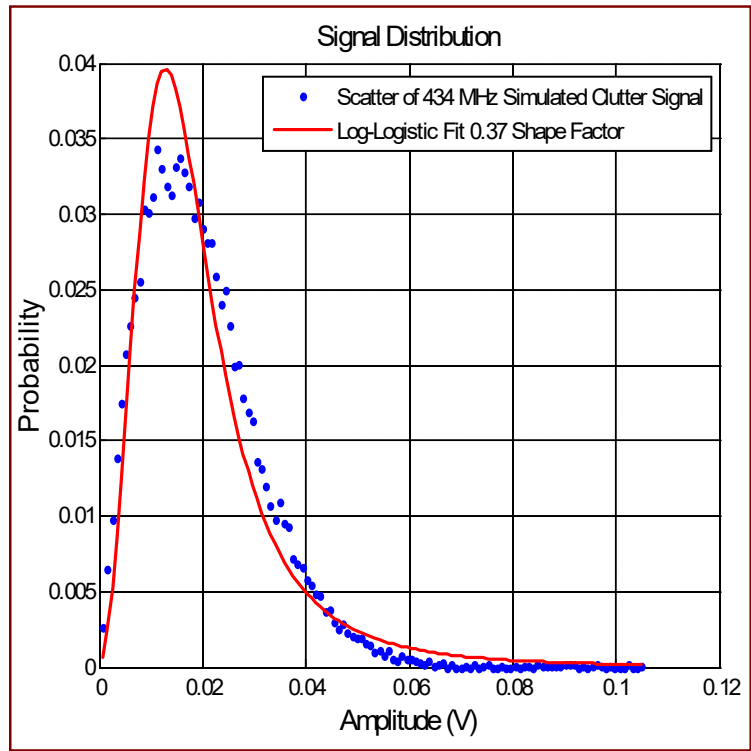

(a)

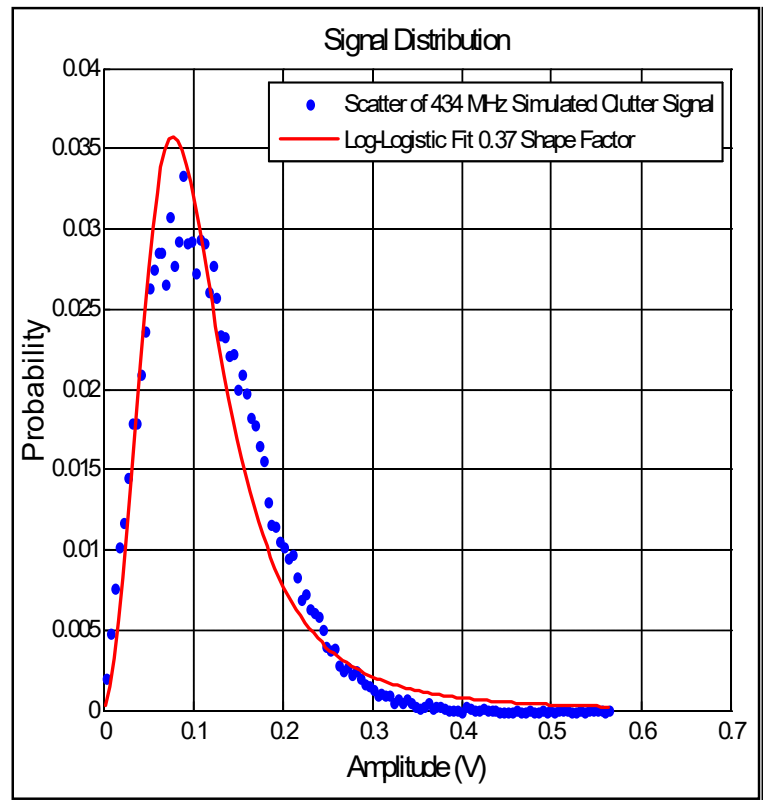

(b)

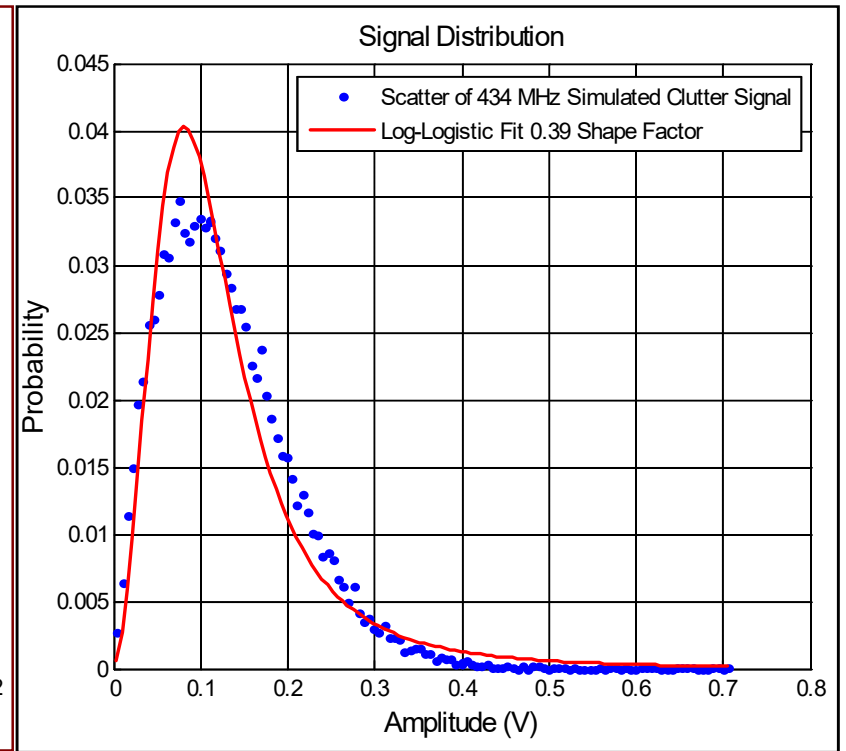

(c)

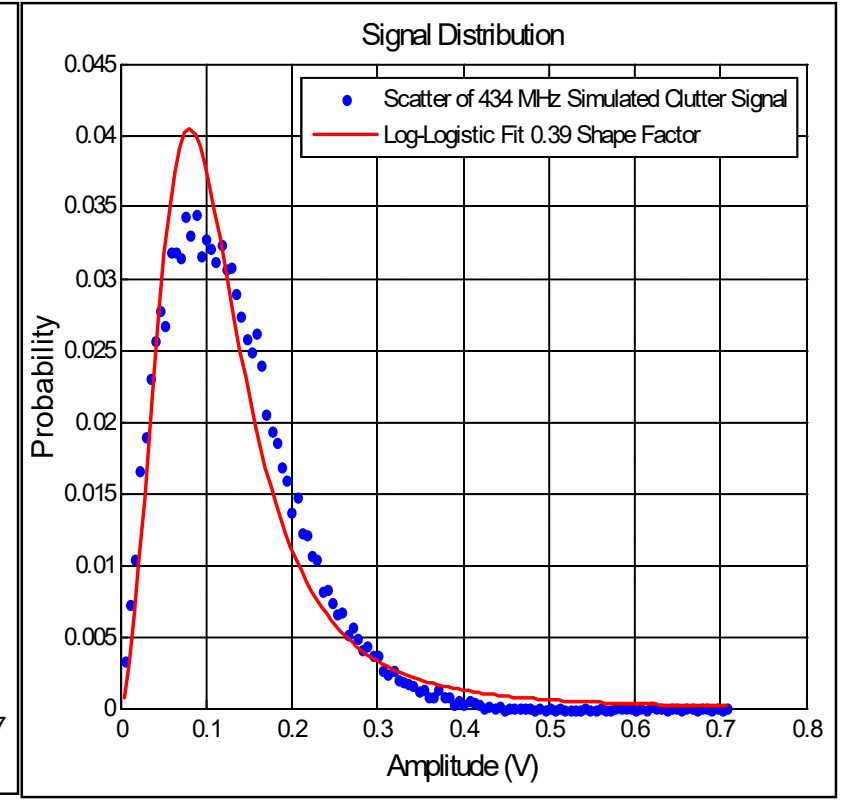

(d)

Fig.5. Signal distribution of simulated clutter signal with number of fit shape factor, (a) low, (b) medium, (c) strong, and (d) very strong 


\subsection{Percentage Error}

Section C and D show the similarity between the measured and simulated clutter signal based on the observation. In this section, we come out with a calculation of the percentage error between the measured and simulated clutter signal in order to evaluate the relevance of our results. In most cases, a percent error or difference of less than $10 \%$ are acceptable. We use root mean square error (RMSE) equation to calculate the percentage error where $i$ is the number of the sample clutter data, $c_{i}$ is the amplitude value for the clutter data and $\hat{c}_{i}$ is the amplitude value for the statistical model.

$\operatorname{RMSE}(\%)=\sqrt{\frac{1}{n} \sum_{i=1}^{n}\left(c_{i}-\hat{c}_{i}\right)^{2}} * 100$

Table 3 shows the calculated percentage error between the measured and simulated clutter signal for low, medium, strong and very strong clutter strength. From the results, it can be seen that the errors are below 10\%, which indicate that the simulation process can be acceptable. Very strong clutter records the highest error, as the clutter signal is difficult to be replicated since the signal varies very strong due to the wind speed during the measurement.

Table 3. Percentage error between measured and simulated cutter signal

\begin{tabular}{cc}
\hline Clutter Strength & Error (\%) \\
\hline Low & 1.50 \\
Medium & 5.70 \\
Strong & 6.45 \\
Very Strong & 7.70 \\
\hline
\end{tabular}

\section{CONCLUSION}

An empirical model for Terengganu seaside clutter signal has been developed for FSR system with $434 \mathrm{MHz}$ using statistical distribution approach. The model is based on the value of standard deviation of measured clutter signal, envelope signal and noise signal, and the maximum and minimum envelope values of the measured clutter signal. The model was validated by comparison of the envelope clutter signal and the number of fit shape factor 
between the measured and the simulated clutter signal. The RMSE method is used in this analysis to evaluate the percentage error between the measured and simulated clutter signal. Based on the validation and evaluation, it can be concluded that the simulation process produces a simulated clutter-like signal as the measured clutter signal.

\section{ACKNOWLEDGEMENTS}

N. N. Ismail would like to express her deepest gratitude to Mr. Ismail Abdullah, Mrs. Maziah Yusof and Mr. Abdul Razak Basarudin for the endless support, aspiring guidance and invaluable assistance in sharing knowledge and ideas.

\section{REFERENCES}

[1] Sizov V, Hu C, Antoniou M, Cherniakov M. Vegetation clutter spectral properties in VHF/UHF bistatic Doppler radar. In IEEE Radar Conference, 2008, pp. 1-6

[2] Anastassopoulos V, Lampropoulos G A. Radar clutter modelling using finite PDF tail. Electronics Letters, 1996, 32(3):256-258

[3] Blasch E P, Hensel M. Fusion of distributions for radar clutter modeling. In IEEE International Conference Information Fusion, 2004, pp. 629-636

[4] Valeyrie N, Garello R, Quellec J M, Chabah M. Study of the modeling of radar sea clutter using the KA distribution and methods for estimating its parameters. International Radar Conference-Surveillance for a Safer World, 2009, pp. 1-6

[5] Zhana W Li Z. Modeling and simulation of ground clutter in the improvement factor test. In 6th IEEE Joint International Information Technology and Artificial Intelligence Conference, 2011, pp. 250-253

[6] Lombardo P, Greco M, Gini F, Farina A, Billingsley J B. Impact of clutter spectra on radar performance prediction. IEEE Transactions on Aerospace and Electronic Systems, 2011, 37(3):1022-1038

[7] Watts S. Radar sea clutter modelling and simulation-Recent progress and future challenges. in IET Seminar on Radar Clutter Modelling, 2008, pp. 1-7

[8] Liang J, Liang Q, Samn S W. Foliage clutter modeling using the UWB radar. In IEEE 
International Conference on Communications, 2008, pp. 1937-1941

[9] Liang J, Liang Q. Outdoor propagation channel modeling in foliage environment. IEEE Transactions on Vehicular Technology, 2010, 59(5):2243-2252

[10] Ismail N N, Rashid N E A, Zakaria N A, Khan Z I. FSR ground clutter distribution model analysis for VHF band. In IET International Radar Conference, 2015, pp. 1-6

[11] Ismail N N, Rashid N E A, Khan Z I, Rashid M F A. Measurement, processing and modeling of a tropical foliage clutter using forward scatter radar micro-sensor network with VHF and UHF bands. In International Conference on Radar, Antenna, Microwave, Electronics and Telecommunications, 2015, pp. 76-81

[12] Ismail N N, Rashid N E A, Khan Z I. Statistical analysis for Terengganu forward scatter radar seaside clutter. Jurnal Teknologi, 2016, 78(5-7):7-14

[13] Afifah T, Nor A Z, Atiqah A R, Mohamad H J, Zairi I R. Variation of VHF/UHF of forward scattering radar due to solar radiation. ARPN Journal of Engineering and Applied Sciences, 2017, 12(10):3278-3284

\section{How to cite this article:}

Ismail NN, Rashid NEA, Ismail Khan Z, Ripin N. Empirical model for terengganu forward scatter radar seaside clutter with uhf band. J. Fundam. Appl. Sci., 2017, 9(3S), 189-198. 